\title{
Evaluación Ciudadana de Competencias Básicas de Lectura y Aritmética y Análisis de Factores Asociados en Yucatán, México
}

\section{Citizen-Led Assessment of Achieve Literacy and Numeracy and Performance Factors Analysis in Yucatán, Mexico}

\author{
Samana Vergara-Lope Tristán *1 \\ Felipe J. Hevia ${ }^{2}$ \\ Víctor Rabay ${ }^{1}$ \\ ${ }^{1}$ Universidad Veracruzana \\ ${ }^{2}$ CIESAS-Centro de Investigaciones y Estudios Superiores en Antropología Social
}

\begin{abstract}
Introducción: No se cuenta con suficiente información respecto del nivel de competencias básicas de lectura y aritmética en el estado de Yucatán, a pesar del desarrollo de las evaluaciones de logro educativo en México. Tampoco existen análisis sobre factores individuales, familiares y sociales asociados a la adquisición de estas competencias en esa entidad. Método: se entrevistaron 1397 sujetos entre 5 y 16 años en una muestra representativa de hogares en Yucatán $(\mathrm{N}=1009)$. Se aplicó el instrumento Medición Independiente de Aprendizajes y una encuesta sobre variables asociadas con tres apartados: 1) datos socioeconómicos y capital cultural educativo; 2) discapacidad y síntomas de salud mental; 3) datos generales y escolares de niños, niñas, adolescentes y preguntas sobre motivación. Resultados: existe alta cobertura escolar, pero se encuentra un porcentaje importante de sujetos con problemas de comprensión inferencial, dificultades en la resolución de problemas matemáticos básicos y uso de operaciones como la resta y la división. Condiciones de discapacidad, motivación escolar, la lengua materna, el capital cultural escolar y acceso a bibliotecas públicas se asocian con estos resultados. Discusión y conclusiones: es necesario implementar políticas específicas para fortalecer las competencias básicas de lectura y aritmética en Yucatán, así como fomentar la medición de estas competencias usando evaluaciones alternativas y ciudadanas como las presentadas aquí.
\end{abstract}

Palabras clave: Evaluación alternativas, Logro académico, Factores asociados, Examen oral, Competencias.

Introduction: We do not have specific information about the level of literacy and numeracy in the state of Yucatan, despite the development of educational evaluation in Mexico and analysis of individual, family and social factors that influence these results in these State were not found. Method: 1397 subjects between 5 and 16 years were interviewed in a representative sample of households in Yucatan $(\mathrm{N}=1009)$. We applied the "Independent Learning Measurement" (MIA); a questionnaire related with demographic and socio-economic data and educational cultural capital; and a disability and mental health symptoms survey, a motivation's questions and a survey related with school behavior. Results: High school coverage exists. There is a significant percentage of subjects with inferential comprehension problems, in solving problems and use basic mathematical operations such as subtraction and division. Similarly, there are individual, family, and social influences, such as motivation and different languages influences. Cultural-School capital and access to public libraries associated significantly with results identified. Discussion and conclusions: it is necessary to implement specific policies to strengthen basic skills of literacy and numeracy in Yucatan, and promote measurement of these competencies using alternative assessments such as Citizen-Led Assessments presented here.

Keywords: Alternative assessment, Academic achievement, Performance factors, Individual influences, Family influences, Social influences, Competences.

*Contacto: samanavergaralope@hotmail.com

issn: 1989-0397

www.rinace.net/riee/

https://revistas.uam.es/riee
Recibido: 13 de octubre de 2016 $1^{\text {a }}$ Evaluación: 23 de noviembre de 2016 $2^{\text {a }}$ Evaluación: 29 de diciembre de 2016 Aceptado: $\quad 9$ de enero de 2017 


\section{Introducción ${ }^{1}$}

Las competencias básicas de lectura y aritmética se consideran aprendizajes fundamentales necesarios para seguir aprendiendo a lo largo de toda la vida (Organización de las Naciones Unidas para la Educación, la Ciencia y la Cultura [UNESCO], 1990) y forman parte de las metas del objetivo de desarrollo sostenible de Naciones Unidas (Programa de Naciones Unidas para el Desarrollo [PNUD], 2015).

En México, el nacimiento y desarrollo del sistema educativo nacional se ha centrado en el fomento de estas competencias en la escuela, hasta el presente, donde forman parte de los denominados "aprendizajes clave" del más reciente modelo educativo (Secretaría de Educación Básica [SEP], 2016a). Sin embargo, a pesar de su importancia, no existe suficiente información respecto a su desarrollo a nivel estatal. Como veremos en el siguiente apartado, existen múltiples evaluaciones nacionales con representatividad estatal que miden el logro curricular esperado al final de primaria y de secundaria, donde se puede inferir el desarrollo de estas competencias, pero cuya presentación hace difícil identificar problemas específicos con la adquisición de éstas.

Por otro lado, diversas instituciones nacionales e internacionales llevan a cabo estudios sistemáticos relacionados con la identificación de factores asociados al logro educativo. Sin embargo, por lo general estos estudios tienen una representatividad nacional, dificultando su uso en contextos tan diversos como los que presenta un país multicultural y pluriétnico como México.

De esta forma, cuando se pretende analizar específicamente el nivel de competencias básicas y sus factores asociados en una entidad federativa específica, existe muy poca información al respecto. Tal es el caso del estado de Yucatán, ubicado en el sureste de México, región que se caracteriza por sus mayores niveles de pobreza, rezago educativo y menores resultados de logro educativo en comparación con el resto del país. Así, según el Consejo Nacional de Evaluación de la Política para el Desarrollo Social (CONEVAL), los estados del sureste mexicano (Puebla, Veracruz, Tabasco, Oaxaca, Chiapas, Yucatán, Campeche y Quintana Roo) se ubicaban entre los estados con mayor porcentaje de pobreza en México, existiendo en Yucatán un 45.9\% de su población en situación de pobreza (CONEVAL, 2015). De igual manera, el Instituto Nacional para la Evaluación de la Educación (INEE), también ubica a esta región como la que tiene el mayor porcentaje de estudiantes con bajo desempeño, estando bajo la media nacional en resultados de logro educativo esperado (INEE, 2016b).

Según el último censo nacional, Yucatán cuenta con 2,097,175 habitantes de los cuales el $29 \%$ de tres años y más afirma hablar una lengua indígena (Instituto Nacional de Estadística y Geografía [INEGI], 2016). Esta entidad en el ciclo escolar 2015-2016 contaba con 429,744 alumnos de educación básica, atendidos por 22,134 docentes en 3309 escuelas (Secretaría de Educación de Yucatán [SEGEY], 2016). La escasa información disponible respecto a las competencias básicas de lectura y aritmética se

1 Este trabajo forma parte del proyecto de investigación "Medición Independiente de Aprendizajes" de CIESAS-Golfo que cuenta con soporte económico de The William \& Flora Hewlett Foundation Grantt 20152650 y del proyecto "Medición Independiente de Aprendizajes-MIA, evaluación de aprendizajes básicos en Veracruz" de la Universidad Veracruzana. 
encuentra en los resultados de las pruebas estandarizadas de respuesta múltiple que han aplicado diversas agencias del estado mexicano desde mediados de la década de 2000. De igual manera, en la bibliografía no se encuentra información específica sobre los factores asociados al bajo nivel educativo identificado en la entidad.

Este artículo tiene por objeto analizar las competencias básicas de lectura y aritmética entre los niños y jóvenes del estado de Yucatán, así como examinar factores individuales, familiares y sociales asociados a estos resultados. Para ello, se utilizaron los datos del proyecto "Medición Independiente de Aprendizajes" (MIA). MIA es una evaluación ciudadana que se enfoca en las competencias básicas de lectura y aritmética. Este proyecto utiliza la metodología de "evaluaciones ciudadanas de aprendizajes" (Citizen Led Assessment) que se llevan a cabo en diversos países de Asia y África hace más de 10 años $\mathrm{y}$ que son reconocidas como evaluaciones pertinentes para medir estas competencias básicas. Las organizaciones que llevan a cabo estas evaluaciones se han reunido en la red PAL (People's Action for Learning) buscando ampliar el uso de esta metodología a más países (PAL Network, 2015). Sus características principales son las siguientes: son organizadas por organizaciones civiles y académicos de manera autónoma a las autoridades educativas, utiliza instrumentos muy simples de medición, buscando que cualquier padre de familia pueda comprender sus resultados, se realiza en hogares, no en escuelas, buscando valorar a los niños que van y que no van a la escuela, se aplica uno a uno a cada niño en su hogar, es decir, no son pruebas de respuesta múltiple, los aplicadores son ciudadanos voluntarios y capacitados, y los resultados de estas evaluaciones buscan involucrar a más actores en la temática educativa (Hevia y Vergara-Lope, 2016; Instituto de Estadística de la UNESCO, 2016; Save the Children, 2013).

Con estos resultados, se pretende aportar argumentos sobre la necesidad de generar políticas educativas específicas para mejorar las competencias básicas, sobre todo en aritmética, y fomentar el uso de este tipo de evaluaciones ciudadanas como una alternativa costo-efectiva que produce información y permite desarrollar intervenciones educativas que subsanen los problemas identificados.

\section{Fundamentación teórica}

\subsection{La evaluación de las competencias básicas de lectura y aritmética en México y Yucatán}

Como en diversos países, la evaluación educativa en México se ha desarrollado de manera creciente en las últimas dos décadas, generando diversos estudios nacionales sobre logro educativo, tanto por parte de la SEP como del INEE. Desde mediados de la década de 2000, primero el INEE con el "Examen para la Calidad y el Logro Educativo" (Excale) y luego la SEP con la "Evaluación Nacional de Logro Académico en Centros Escolares" (Enlace), se ha intentado evaluar el logro educativo, basándose en los aprendizajes curriculares esperados. Estas evaluaciones compartían algunos aspectos metodológicos, como ser pruebas de opción múltiple que se aplican en las escuelas y presentar los resultados por niveles de logro, pero diferían en diversos aspectos, entre los que destacaban el carácter censal de Enlace (se aplicaba a todos los niños entre 30 de primaria y 30 de secundaria) y muestral de Excale (donde se aplicaba a una muestra 
representativa de escuelas y grados escolares) (Backhoff y Contreras Roldán, 2014, INEE, 2016a; SEP, 2011).

En 2013 se llevó a cabo una reforma educativa que implicó, entre otras cosas, la reestructuración del sistema de evaluación. Esto implicó la creación del "Plan Nacional para la Evaluación de los Aprendizajes" (PLANEA), la estrategia nacional para la medir los resultados de logro educativo en el aula, desarrollado por el INEE y aplicado en una muestra representativa de alumnos de un censo de escuelas en todo el país. Esta prueba se aplica en $6^{\circ}$ de primaria y $3^{\circ}$ de secundaria y tiene como propósito conocer la medida en que los estudiantes logran el dominio de un conjunto de aprendizajes esenciales en diferentes momentos de la educación obligatoria en México (INEE, 2015).

Los resultados de estas pruebas para el sureste mexicano en general, y para Yucatán en particular son consistentes y muestran bajo nivel de logro educativo según los aprendizajes curriculares esperados. Pero estos resultados dicen poco sobre las competencias básicas de lectura y aritmética. Como se puede ver en las siguientes tablas, entre 2006 y 2013 la mayoría de los niños y niñas de primaria y secundaria en Yucatán se encontraron en los niveles "insuficiente" y "elemental", tanto en matemáticas como en español.

Tabla 1. Porcentaje de resultado prueba Enlace Yucatán primaria español y matemáticas, 2006-2013

\begin{tabular}{lccccc}
\hline PRIMARIA YUCATÁN & AÑO & INSUFICIENTE & ELEMENTAL & BUENO & EXCELENTE \\
\hline \multirow{5}{*}{ Español } & 2006 & 20,6 & 60,7 & 17,2 & 1,4 \\
& 2007 & 18,9 & 57,7 & 20,7 & 2,7 \\
& 2008 & 20,6 & 50,0 & 25,8 & 3,6 \\
& 2009 & 17,3 & 50,3 & 27,6 & 4,8 \\
& 2010 & 16,3 & 47,7 & 29,8 & 6,2 \\
& 2011 & 15,5 & 46,8 & 30,1 & 7,5 \\
& 2012 & 14,9 & 45,4 & 29,4 & 10,3 \\
Matemáticas & 2013 & 12,5 & 44,5 & 32,8 & 10,1 \\
& 2006 & 22,7 & 64,4 & 11,9 & 1,1 \\
& 2007 & 20,4 & 61,1 & 16,1 & 2,4 \\
& 2008 & 24,0 & 52,0 & 20,3 & 3,7 \\
& 2009 & 20,5 & 52,1 & 22,6 & 4,8 \\
& 2010 & 20,1 & 49,4 & 23,8 & 6,7 \\
& 2011 & 18,2 & 50,9 & 22,8 & 8,1 \\
& 2012 & 13,7 & 46,5 & 25,8 & 14,1 \\
& 2013 & 12,8 & 40,8 & 28,1 & 18,2 \\
\hline
\end{tabular}

Fuente: Elaboración propia sobre (SEP, 2016b).

Algo similar se puede observar en los resultados de la prueba Excale. La última vez que se aplicó en Yucatán fue en 2012 a estudiantes de secundaria. Así, el único dato agregado que se reporta es el porcentaje de alumnos en nivel "insuficiente", que fue de $19 \%$ en español y de $29 \%$ en matemáticas (INEE, 2013).

De igual manera, los resultados de Planea se presentan en cuatro niveles: el nivel I significa "Logro Insuficiente", el nivel II es "Logro apenas indispensable", el nivel tres es "Logro satisfactorio" y finalmente el nivel IV es "Sobresaliente". La siguiente tabla muestra que el $49,6 \%$ de sexto de primaria y el $28,1 \%$ de tercero de secundaria están en el nivel I para el campo de comunicación y lenguaje y el $63,1 \%$ y $64,9 \%$ respectivamente para el campo de matemáticas. 
Tabla 2. Porcentajes de resultado prueba Enlace Yucatán, secundaria español y matemáticas, 2006-2013

\begin{tabular}{lccccc}
\hline SECUNDARIA YUCATÁN & AÑO & INSUFICIENTE & ELEMENTAL & BUENO & EXCELENTE \\
\hline \multirow{5}{*}{ Español } & 2006 & 35,1 & 48,6 & 15,3 & 1,0 \\
& 2007 & 35,8 & 45,6 & 17,6 & 1,0 \\
& 2008 & 32,3 & 50,4 & 16,6 & 0,7 \\
& 2009 & 27,7 & 52,0 & 19,6 & 0,7 \\
& 2010 & 36,5 & 45,4 & 17,2 & 0,9 \\
& 2011 & 36,1 & 45,7 & 17,3 & 0,9 \\
& 2012 & 32,6 & 44,3 & 21,2 & 1,9 \\
& 2013 & 32,4 & 46,9 & 19,4 & 1,4 \\
\hline \multirow{5}{*}{ Matemáticas } & 2006 & 60,3 & 35,0 & 4,2 & 0,4 \\
& 2007 & 57,2 & 37,3 & 5,1 & 0,4 \\
& 2008 & 55,0 & 36,0 & 8,1 & 0,9 \\
& 2009 & 55,1 & 35,2 & 8,6 & 1,0 \\
& 2010 & 53,5 & 35,2 & 9,5 & 1,8 \\
& 2011 & 52,8 & 32,4 & 11,4 & 3,4 \\
& 2012 & 47,9 & 32,6 & 13,8 & 5,7 \\
\hline
\end{tabular}

Fuente: Elaboración propia sobre (SEP, 2016b).

Tabla 3. Porcentajes por nivel de logro en comunicación y lenguaje y matemáticas primaria y secundaria para Yucatán, PLANEA 2015

\begin{tabular}{lcccccc}
\hline CAMPOS FORMATIVOS & \multirow{2}{*}{ GRADO } & ENTIDAD & NIVEL 1 & NIVEL 2 & NIVEL 3 & NIVEL 4 \\
\hline \multirow{2}{*}{ Comunicación y lenguaje } & \multirow{2}{*}{6 primaria } & Yucatán & 49,6 & 34,2 & 13,8 & 2,3 \\
& & País & 49,5 & 33,2 & 14,6 & 2,6 \\
\hline \multirow{2}{*}{ Matemáticas } & \multirow{2}{*}{6 primaria } & Yucatán & 63,1 & 18,9 & 13 & 5 \\
& & País & 60,5 & 18,9 & 13,8 & 6,8 \\
\hline \multirow{2}{*}{ Comunicación y lenguaje } & \multirow{2}{*}{3 secundaria } & Yucatán & 28,1 & 46,9 & 18,6 & 6,4 \\
& & País & 29,4 & 46 & 18,4 & 6,1 \\
\hline \multirow{2}{*}{ Matemáticas } & \multirow{2}{*}{3 secundaria } & Yucatán & 64,9 & 23,8 & 7,9 & 3,4 \\
& & País & 65,4 & 24 & 7,5 & 3,1 \\
\hline
\end{tabular}

Fuente: Elaboración propia sobre (INEE, 2015).

Estas pruebas representan avances fundamentales para la evaluación educativa por su solidez técnica y por el creciente uso que tienen para discutir de política educativa. Sin embargo, comparten una serie de dificultades de comunicación que dificultan su uso para mejorar el nivel educativo y analizar las competencias básicas de lectura y aritméticas, y que son comunes a este tipo de evaluación educativa de gran escala (McLauchlan, 2009). En primer lugar, la utilización de categorías genéricas (como “insuficiente" o "nivel 1") ayuda a tener un panorama general sobre las deficiencias estructurales del sistema, pero resulta menos útil la información así entregada para comprender el estado específico de los aprendizajes de los estudiantes, pues a partir de estas variables tanto la sociedad civil como el cuerpo docente no tiene forma de entender cuáles son las habilidades básicas particulares que pueden o no realizar los alumnos. En segundo lugar, estas pruebas sólo consideran a la población que acude a la escuela, excluyendo de la medición a todos los niños que por múltiples circunstancias no asisten a la escuela, dejando de lado a esta población de las mediciones educativas (Instituto de Estadística de la UNESCO, 2016). En tercer lugar, las pruebas se aplican por lo general a cursos terminales de primaria y secundaria (como es el caso de la encuesta nacional de Planea), lo que representa información más útil para la planeación educativa que para la acción formativa en las 
escuelas. En este sentido, con la forma de comunicar estos resultados, es difícil para un padre de familia identificar el nivel de logro educativo en competencias básicas como leer o saber resolver operaciones aritméticas sencillas. En cuarto lugar, estas pruebas son de opción múltiple, no pudiendo aprovechar ciertas ventajas comparativas que tienen las pruebas orales para evaluar lectura (Instituto de Estadística de la UNESCO, 2016). Otra limitación tiene relación con el potencial uso punitivo de este tipo de evaluaciones, ya sea para castigar o premiar a docentes, más que para generar evaluaciones formativas que permitan un llamado a la acción para transformar estos resultados (Martínez Rizo, 2009).

Por esto, resulta pertinente generar otro tipo de evaluaciones de logro educativo. En este rubro sobresale la experiencia de las denominadas evaluaciones ciudadanas de aprendizajes (Citizen Led Assessment) que se han aplicado en diversos países en vías de desarrollo de África, Asia y América Latina y que son reconocidas como experiencias valiosas para fomentar la evaluación formativa de competencias básicas de lectura y aritmética así como para fomentar la participación ciudadana en la educación en las temáticas de evaluación educativa (Banerji, Bhattacharjea y Wadhwa, 2013; Report, 2016).

Estas evaluaciones ciudadanas se pueden considerar alternativas porque son organizadas e implementadas por organizaciones civiles, y en algunos casos, académicas. Así, por ejemplo, en India, Pakistán, Mali, Uganda, Kenia y Tanzania son organizadas por organizaciones no gubernamentales, mientras que en el caso de México y Senegal son centros universitarios los responsables de su implementación. Esto implica, por un lado, que los objetivos de estas evaluaciones (el para quê) buscan alinearse con demandas ciudadanas específicas de cada país donde se llevan a cabo. En el caso de India, por ejemplo, la organización civil Pratham generó esta metodología, en primer lugar, para poder valorar sus propias intervenciones extra-escolares relacionadas con alfabetización (Banerji, Bhattacharjea y Wadhwa, 2013), mientras que en México los objetivos de las evaluaciones del proyecto MIA tienen que ver con incrementar la participación social en educación y posicionar la discusión sobre los aprendizajes en el contexto de reformas educativas más orientadas a políticas de evaluación docente (Vergara-Lope y Hevia, 2016).

Por otro lado, la organización ciudadana se refleja en los procesos de implementación: cada ejercicio de evaluación ciudadana implica la participación de diversas organizaciones civiles y académicas en los territorios donde se lleva a cabo. Estas organizaciones permiten identificar y reclutar a centenas de ciudadanos voluntarios se capacitan para levantar la información, buscando así involucrarlos en la problemática de la educación. La información que se genera por medio de este proceso es compartida en los territorios con la organización de talleres donde se presentan los resultados y se buscan respuestas alternativas (Vergara-Lope y Hevia, 2016). Este involucramiento permite la generación de innovaciones educativas orientadas a incrementar las capacidades básicas de lectura, como es el caso del programa Read India que se lleva a cabo por Pratham (Dutt, Kwauk y Robinson, 2016).

En segundo lugar, estas evaluaciones emplean instrumentos sencillos de aplicar y de usar, que requieren ser aplicados niño por niño. Esto significa que no son exámenes de respuesta múltiple, como la mayoría de las evaluaciones masivas que se aplican en 
México. En tercer lugar, porque se aplican en hogares, no en escuelas, buscando, por un lado, medir tanto a los niños que van a la escuela como a los niños que por diversas razones no asisten y, por otro lado, para identificar factores extra-escolares que puedan estar asociados a los aprendizajes. Como veremos, estas encuestas se aplican todos los niños entre 5 y 16 años que vivan en los hogares seleccionados sobre una muestra representativa. Por último, estas pruebas aplican el mismo instrumento a todos los niños, esperando que aquellos sujetos de 5 años no puedan completar el instrumento, pero los de 16 años no tengan problemas en resolverlo.

\subsection{Factores asociados a los aprendizajes}

Junto con la escasez de información respecto al desarrollo de competencias básicas en Yucatán, en la literatura se advierte un déficit de estudios sobre factores asociados al logro académico en esta entidad federativa. En la bibliografía es posible identificar investigaciones relacionadas con los aprendizajes enfocadas temáticamente. Así, por ejemplo, podemos encontrar referencias respecto a problemas comunes a la educación. Es el caso del bullying (Rocha y Espejel, 2008), la deserción (Barroso-Tanoira, 2014), la ansiedad y autoestima en estudiantes (Couoh Lope, Góngora, García Rivero, Macías Aguilar y Olmos Barragán, 2015). Otra área importante de investigación se centra en problemas específicos relacionados con la población maya. Por un lado, desarrollo de la lecto-escritura en mayahablantes (Azuara y Reyes 2011; Mier y Terán y Rabell 2013; Sima, Perales, y Be Ramirez 2014), por otro lado, currículo y educación intercultural (Mijangos, Soberanis y Negrón, 2011); deserción en niños mayas (Mijangos-Noh y Cardos-Dzul, 2011); y condiciones generales de la población rural (Méndez-González, 2010; Stearns, 1986). Sin embargo, estas investigaciones no entregan datos específicos sobre el nivel de aprendizajes en los niños/as yucatecos ni se preguntan sobre factores asociados.

La bibliografía sobre factores asociados al logro educativo es muy extensa. Factores sociales, como el nivel socioeconómico, se han estudiado en múltiples contextos: tanto la relación ingreso-desempeño escolar (Hanushek y Luque, 2002), como las diferencias económicas al interior de los sistemas educativos (Coleman et al., 1966). Recientemente se ha buscado identificar el peso del nivel socioeconómico separando aspecto económico, social y cultural (Hernández Padilla y González, 2011), así como incluir el peso de las desigualdades económicas (Blanco, 2013; Duru-Bellat, 2004).

Un segundo tipo de factores asociados tienen que ver con características individuales de los estudiantes. Con respecto al género (Cervini y Dari, 2009), algunos estudios reportan que las mujeres presentan mejor rendimiento en comprensión lectora mientras que los hombres muestran mejor rendimiento en matemáticas (Carvallo Pontón, Caso y Contreras, 2007). Las condiciones de discapacidad o problemas crónicos de salud han sido relacionados con logros de aprendizaje inferiores (Bravo-Sanzana y Salvo, 2015). En la literatura se ha reportado de manera amplia el vínculo entre la motivación por la escuela y el logro educativo (Ames, 1992; Zenorini, Santos y Monteiro, 2011). Los problemas de salud han sido parte de los factores individuales relacionados con el rendimiento escolar, específicamente los problemas de salud mental (Jiménez y Valle, 2013), sin embargo, sobre esta relación la literatura aún es escasa en América Latina. Existe creciente evidencia respecto a que la interacción en la escuela, y en los espacios de aprendizaje exige a cada niño y niña poner en práctica herramientas que ha adquirido en 
sus diferentes contextos y ambientes, y el uso de estas se verá reflejado en sus esquemas cognitivos y afectivos que utilizará en sus conductas saludables o disfuncionales, si estas conductas resultan ser disfuncionales o desadaptativas funcionarán como conductas de riesgo para su salud mental. A su vez, se expresarán en dificultades sociales y de regulación emocional, así como en problemas en el logro académico (Bravo-Sanzana y Salvo, 2015).

De igual manera, es posible identificar factores familiares como los que se integran bajo el concepto de "capital cultural escolar", donde se incluye la escolaridad de los padres, el acceso a ciertos bienes culturales, y las expectativas de los padres hacia sus hijos (Backhoff, Bouzas, Contreras, Hernández y García, 2007; Hernández Padilla y González, 2011). Dentro de estos factores familiares se puede incluir como un factor fundamental para el caso de América Latina la existencia de una lengua materna diferente del español (UNESCO, 2015). Sin embargo, como ya se dijo, no se cuenta con información específica para el caso de Yucatán sobre cómo estos factores están influyen en la adquisición de las capacidades básicas.

\section{Método}

El diseño de la investigación corresponde a un estudio descriptivo de poblaciones mediante encuestas con muestras probabilísticas de tipo transversal (Montero y León, 2007).

\subsection{Participantes}

En esta investigación participaron 1397 sujetos entre 5 y 16 años, entrevistados en 1009 hogares. La media de edad de los niños y jóvenes entrevistados fue de 9,79 años $(\mathrm{DE}=3,21)$, de los cuales $49,7 \%$ fueron mujeres y 50,3\% hombres. El $98 \%$ de la muestra afirmó asistir regularmente a la escuela. El 86,5\% ( $\mathrm{N}=1144)$ asistía en turno matutino y el 12,9\% ( $\mathrm{N}=170)$ vespertino. Los grados escolares a los que pertenecían fueron se muestran em la tabla 4.

Tabla 4. Grado escolar de la muestra de investigación

\begin{tabular}{lcc}
\hline \multicolumn{1}{c}{ GRADO } & FRECUENCIA & PORCENTAJE \\
\hline No asiste a la escuela & 10 & 0,7 \\
3 preescolar & 120 & 8,6 \\
1 primaria & 156 & 11,2 \\
2 primaria & 162 & 11,6 \\
3 primaria & 145 & 10,4 \\
4 primaria & 150 & 10,7 \\
5 primaria & 142 & 10,2 \\
6 primaria & 122 & 8,7 \\
1 secundaria & 119 & 8,5 \\
2 secundaria & 111 & 7,9 \\
3 secundaria & 72 & 5,2 \\
Preparatoria/bachillerato & 88 & 6,3 \\
\hline Total & 1397 & 100,0 \\
\hline
\end{tabular}

Fuente: Elaboración propia con resultados de la investigación. 
El 93,6\% (N=1203) afirmó asistir a la escuela pública y solo el 6,1\% $(\mathrm{N}=78)$ a escuela privada. De igual manera, el 96,8\% ( $\mathrm{N}=1305)$ asistió a preescolar o kínder, pero solo el $24,4 \%(\mathrm{~N}=322)$ asistió a guardería o estancia escolar antes de preescolar.

El rango de edad seleccionado (5-16 años) responde a fines de comparabilidad con otras mediciones similares realizadas en Asia y África agrupadas en la red PAL (PAL Network, 2015), y permite medir también cobertura escolar para el $3^{\circ}$ de preescolar para el caso mexicano.

\subsection{Muestreo}

Se realizó un muestreo representativo polietápico, probabilístico, estratificado, por conglomerados (Hernández, Fernández y Baptista, 2001; Kerlinger y Lee, 2002), con un $95 \%$ de confianza y un margen de error de $+/-4 \%$. El procedimiento de muestreo implicó tres pasos: 1) selección de conglomerados o unidades primarias de muestreo (UPM), mediante un muestreo aleatorio estratificado, sistemático y con probabilidad proporcional al tamaño; 2) selección de manzanas al azar dentro del conglomerado y; 3) selección sistemática de viviendas en las Manzanas. Se entrevistaron a todos los niños y niñas de entre 5 y 16 años que vivían en el hogar seleccionado y que quisieron participar en el estudio. Las unidades primarias fueron las secciones electorales, pertenecientes a cinco distritos electorales de Yucatán. La información se levantó en 79 localidades urbanas y rurales en 15 municipios diferentes. La aplicación se realizó por voluntarios que recibieron una capacitación previa de seis horas.

\subsection{Instrumentos}

Se utilizaron las tres formas paralelas de los instrumentos MIA para lectura y matemáticas (Hevia y Vergara-Lope, 2016). Cada versión consta de 10 reactivos que corresponden a un nivel curricular de segundo de primaria, para el caso de lectura, y cuarto de primaria, para el caso de matemáticas. Los índices de consistencia interna totales van de 0,81 a 0,86 , para las tres versiones, y coeficientes de equivalencia de 0,88 a 0.93 entre las tres pruebas paralelas. El instrumento de lectura consta de cinco reactivos: sílaba, palabra, enunciado, historia y comprensión. El instrumento de matemáticas contiene: identificación de números 10-99, suma de decenas con acarreo, resta de decenas con acarreo, división de centenas y resolución de un problema que implica el uso de dos operaciones.

Además de los instrumentos para medir aprendizajes básicos, se aplicó una encuesta de factores asociados al desempeño académico, que incluyó los siguientes tres apartados. 1) Apartado dirigido a padres, con preguntas sobre capital cultural educativo como escolaridad de los padres, acceso a bienes de consumo cultural como libros, internet y cine, y expectativas de estudio de los hijos (Backhoff, 2011; Backhoff et al., 2007). 2) Apartado dirigido a padres con preguntas relacionadas a cada uno de los niños. Este apartado contiene en primer lugar preguntas sobre condiciones de discapacidad presentes en los niños. Estas preguntas se tomaron del módulo IX de salud de la Encuesta de Características Socioeconómicas de los Hogares 2014, del Programa de Desarrollo Humano Oportunidades (Sedesol, 2014). En segundo lugar, se incluyó el Cuestionario de Reporte para Niños (Reporting Questionnaire for Children: RQC) (Gómez Espinoza, Rico Diaz, Caraveo Anduaga y Guerrero Cansino, 1993) que consta de 10 preguntas que miden signos y síntomas de salud mental infantil. Este instrumento es de 
tamizaje en salud mental y ayuda a evaluar la posible presencia de problemas en esta área, no se puede utilizar para diagnosticar un trastorno mental. Una respuesta positiva o más, indica que se debe de prestar más atención al caso para un futuro diagnóstico y tratamiento. Es aplicable a los padres o cualquier adulto que tenga una relación cercana con el niño o niña. Utiliza un punto de corte $0 / 1$ y tiene sensibilidad de $87,2 \%$, especificidad de $73,9 \%$, valor predictivo positivo de $69,4 \%$, valor predictivo negativo de $89,5 \%$ y tasa de clasificación errónea de 20,7\%. Por último, 3) apartado dirigido a niños, niñas y adolescentes en donde se les preguntaba, además de su edad y escolaridad, su posición entre los hermanos, el nombre de su escuela, el turno, y preguntas sobre motivación escolar y dinámicas al interior de la escuela.

\subsection{Procedimientos}

Los instrumentos fueron aplicados en los hogares por 150 ciudadanos voluntarios previamente capacitados, que en su mayoría eran estudiantes universitarios de cursos superiores. La información se recolectó en el mes de noviembre de 2015. La supervisión de la aplicación y monitoreo de los aplicadores estuvo a cargo de un servicio de monitoreo externo.

La información se procesó en una base de datos en el programa SPSS, y se sometió a una serie de procedimientos de estadística descriptiva, paramétrica y no paramétrica (Hernández et al., 2001).

\section{Resultados}

Los resultados se presentan en dos secciones. En la primera se muestran los datos de lectura y aritmética a los que llegaron según el instrumento MIA, y en la segunda parte se discuten algunos factores asociados identificados.

\subsection{Resultados de lectura y aritmética}

En la tabla 5 se presentan los porcentajes de los niveles de lectura alcanzados por los participantes. Los sujetos que no pudieron identificar sílabas (nivel "ninguno") disminuye de $56,5 \%$ en preescolar a $4,2 \%$ en tercero de primaria, mientras que los que pudieron responder correctamente la pregunta de comprensión aumenta de $5,2 \%$ en primero de primaria al $70 \%$ en sexto de primaria.

Tabla 5. Porcentajes de nivel le lectura por grado escolar, instrumento MIA, Yucatán

\begin{tabular}{lcccccc}
\hline & NINGÚN & SílABA & PALABRA & ORACIÓN & HISTORIA & COMPRENSIÓN \\
\hline 3 preescolar & $56,8 \%$ & $22,0 \%$ & $8,5 \%$ & $6,8 \%$ & $1,7 \%$ & $4,2 \%$ \\
1 primaria & $33,5 \%$ & $32,3 \%$ & $15,5 \%$ & $10,3 \%$ & $3,2 \%$ & $5,2 \%$ \\
2 primaria & $5,0 \%$ & $15,0 \%$ & $25,6 \%$ & $20,0 \%$ & $19,4 \%$ & $15,0 \%$ \\
3 primaria & $4,2 \%$ & $4,2 \%$ & $9,0 \%$ & $22,2 \%$ & $22,9 \%$ & $37,5 \%$ \\
4 primaria & $0,7 \%$ & $4,8 \%$ & $6,8 \%$ & $18,4 \%$ & $25,2 \%$ & $44,2 \%$ \\
5 primaria & & $1,4 \%$ & $4,9 \%$ & $11,3 \%$ & $23,2 \%$ & $59,2 \%$ \\
6 primaria & $0,8 \%$ & $1,7 \%$ & $2,5 \%$ & $8,3 \%$ & $16,5 \%$ & $70,2 \%$ \\
1 secundaria & & & $0,8 \%$ & $3,4 \%$ & $16,9 \%$ & $78,8 \%$ \\
2 secundaria & & & & $1,8 \%$ & $10,9 \%$ & $87,3 \%$ \\
3 secundaria & & $1,4 \%$ & & $1,4 \%$ & $9,7 \%$ & $87,5 \%$ \\
Bachillerato & & & & $1,1 \%$ & $9,1 \%$ & $89,8 \%$ \\
\hline Total & $10,0 \%$ & $8,5 \%$ & $7,9 \%$ & $10,8 \%$ & $15,2 \%$ & $47,6 \%$ \\
\hline
\end{tabular}

Fuente: Elaboración propia sobre resultados de la investigación. 
La siguiente figura presenta de manera gráfica los resultados de la tabla, donde se advierten el primer hallazgo principal: un importante porcentaje de sujetos en todos los grados no pudieron responder una pregunta de comprensión simple (que corresponde a segundo de primaria).

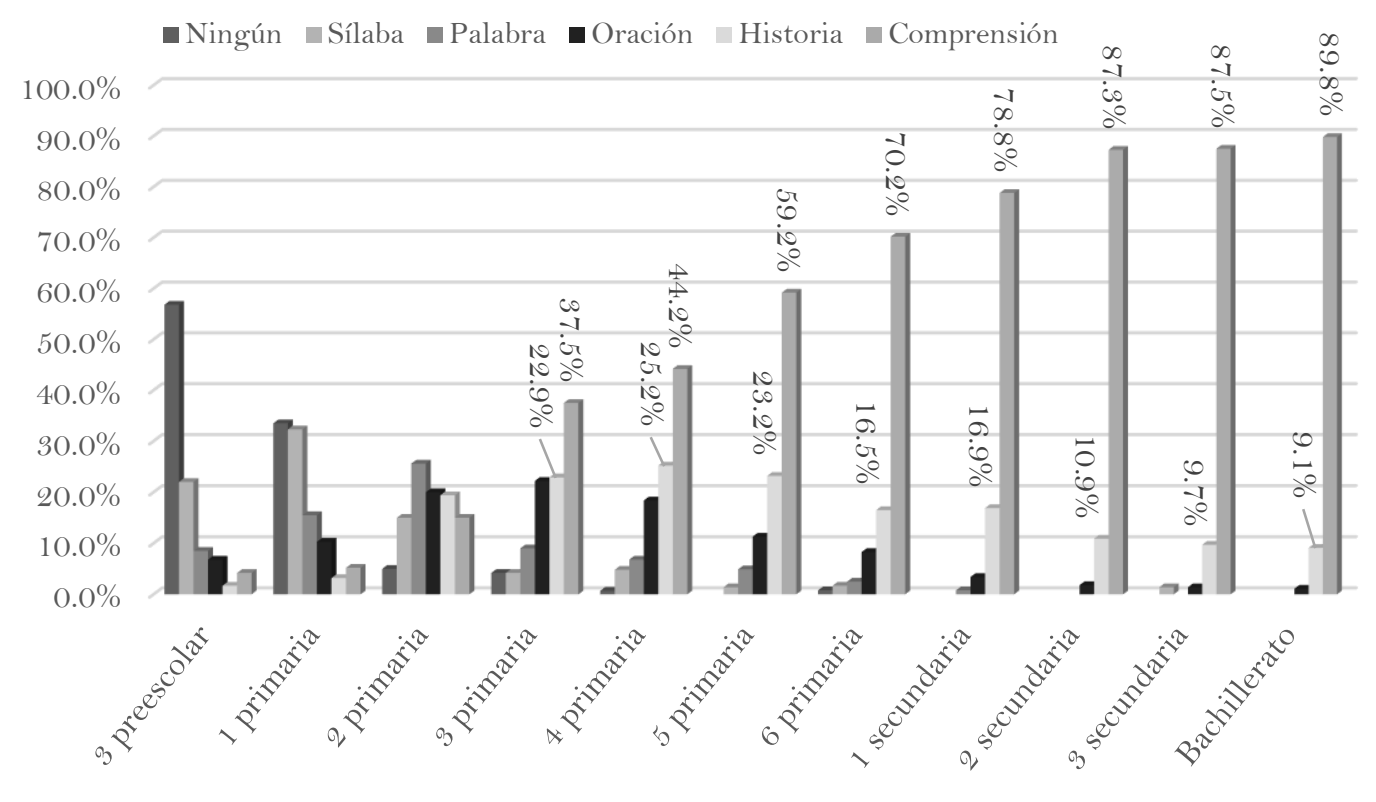

Figura 1. Porcentajes de lectura por grado escolar, Yucatán Fuente: Elaboración propia.

Así, la figura 1 muestra cómo en secundaria hay un 30\% de sujetos de sexto de primaria que tienen problemas al responder una pregunta de comprensión, lo mismo que el 13\% en promedio de sujetos de secundaria.

La tabla 6 muestra los resultados de lectura agregados. Esto permite ver que en sexto de primaria el 13\% sujetos no pudo leer una historia simple, lo mismo pasó con casi del 40\% de los sujetos de cuarto. Es decir, un segundo hallazgo muestra que un porcentaje importante de niños de los últimos grados de primaria tienen problemas para leer una historia simple.

Tabla 6. Porcentajes de sujetos que lograron hacer correctamente cada uno de los niveles de lectura instrumento MIA. Yucatán

\begin{tabular}{|c|c|c|c|c|c|}
\hline & SílABA & PALABRA & ORACIÓN & HISTORIA & COMPRENSIÓN \\
\hline 3 preescolar & $43,2 \%$ & $21,2 \%$ & $12,7 \%$ & $5,9 \%$ & $4,2 \%$ \\
\hline 1 primaria & $66,5 \%$ & $34,2 \%$ & $18,7 \%$ & $8,4 \%$ & $5,2 \%$ \\
\hline 2 primaria & $95,0 \%$ & $80,0 \%$ & $54,4 \%$ & $34,4 \%$ & $15,0 \%$ \\
\hline 3 primaria & $95,8 \%$ & $91,6 \%$ & $82,6 \%$ & $60,4 \%$ & $37,5 \%$ \\
\hline 4 primaria & $99,4 \%$ & $94,6 \%$ & $87,8 \%$ & $69,4 \%$ & $44,2 \%$ \\
\hline 5 primaria & $100,0 \%$ & $98,6 \%$ & $93,7 \%$ & $82,4 \%$ & $59,2 \%$ \\
\hline 6 primaria & $99,2 \%$ & $97,5 \%$ & $95,0 \%$ & $86,7 \%$ & $70,2 \%$ \\
\hline 1 secundaria & $99,9 \%$ & $99,9 \%$ & $99,1 \%$ & $95,7 \%$ & $78,8 \%$ \\
\hline 2 secundaria & $100,0 \%$ & $100,0 \%$ & $100,0 \%$ & $98,2 \%$ & $87,3 \%$ \\
\hline 3 secundaria & $100,0 \%$ & $98,6 \%$ & $98,6 \%$ & $97,2 \%$ & $87,5 \%$ \\
\hline Bachillerato & $100,0 \%$ & $100,0 \%$ & $100,0 \%$ & $98,9 \%$ & $89,8 \%$ \\
\hline
\end{tabular}

Fuente: Elaboración propia sobre resultados de la investigación. 
Respecto a los resultados de aritmética, se puede apreciar limitaciones importantes tanto en la resolución de operaciones como resta y división, como en su aplicación para la resolución de un problema que requiere dos operaciones aritméticas. La tabla 7 muestra los resultados por grado escolar, donde es posible ver cómo disminuye el "ningún" hasta tercero de primaria y cómo aumenta el porcentaje de resolución de "problema” a partir de sexto de primaria.

Tabla 7. Porcentajes de nivel de aritmética por grado escolar instrumento MIA, Yucatán

\begin{tabular}{|c|c|c|c|c|c|c|}
\hline & NINGÚN & NÚMERO 10-99 & SUMA & RESTA & DIVISIÓN & PROBLEMA \\
\hline 3 preescolar & $60,8 \%$ & $28,3 \%$ & $3,3 \%$ & $4,2 \%$ & & $3,3 \%$ \\
\hline 1 primaria & $42,3 \%$ & $40,4 \%$ & $7,1 \%$ & $9,0 \%$ & & $1,3 \%$ \\
\hline 2 primaria & $8,0 \%$ & $37,0 \%$ & $28,4 \%$ & $24,7 \%$ & $1,2 \%$ & $0,6 \%$ \\
\hline 3 primaria & $4,1 \%$ & $10,3 \%$ & $35,2 \%$ & $40,0 \%$ & $8,3 \%$ & $2,1 \%$ \\
\hline 4 primaria & $1,3 \%$ & $10,7 \%$ & $22,7 \%$ & $36,0 \%$ & $22,7 \%$ & $6,7 \%$ \\
\hline 5 primaria & $0,7 \%$ & $7,0 \%$ & $23,9 \%$ & $27,5 \%$ & $33,8 \%$ & $7,0 \%$ \\
\hline 6 primaria & & $2,5 \%$ & $18,9 \%$ & $23,8 \%$ & $32,8 \%$ & $22,1 \%$ \\
\hline 1 secundaria & & $7,6 \%$ & $13,4 \%$ & $16,0 \%$ & $36,1 \%$ & $26,9 \%$ \\
\hline 2 secundaria & & $2,7 \%$ & $15,3 \%$ & $18,9 \%$ & $28,8 \%$ & $34,2 \%$ \\
\hline 3 secundaria & & $2,8 \%$ & $13,9 \%$ & $18,1 \%$ & $22,2 \%$ & $43,1 \%$ \\
\hline Bachillerato & $1,1 \%$ & $6,8 \%$ & $12,5 \%$ & $13,6 \%$ & $13,6 \%$ & $52,3 \%$ \\
\hline
\end{tabular}

Fuente: Elaboración propia sobre resultados de la investigación.

Al graficar estos resultados, se puede constatar el bajo nivel de resolución de problemas en todos los grados, donde solo bachillerato supera levemente el 50\%. Considerando que es un problema de complejidad de cuarto grado, es particularmente bajo el nivel alcanzado por los últimos grados de primaria, donde solo el $7 \%$ de quinto grado y el $22 \%$ de sexto pudo resolverlo.

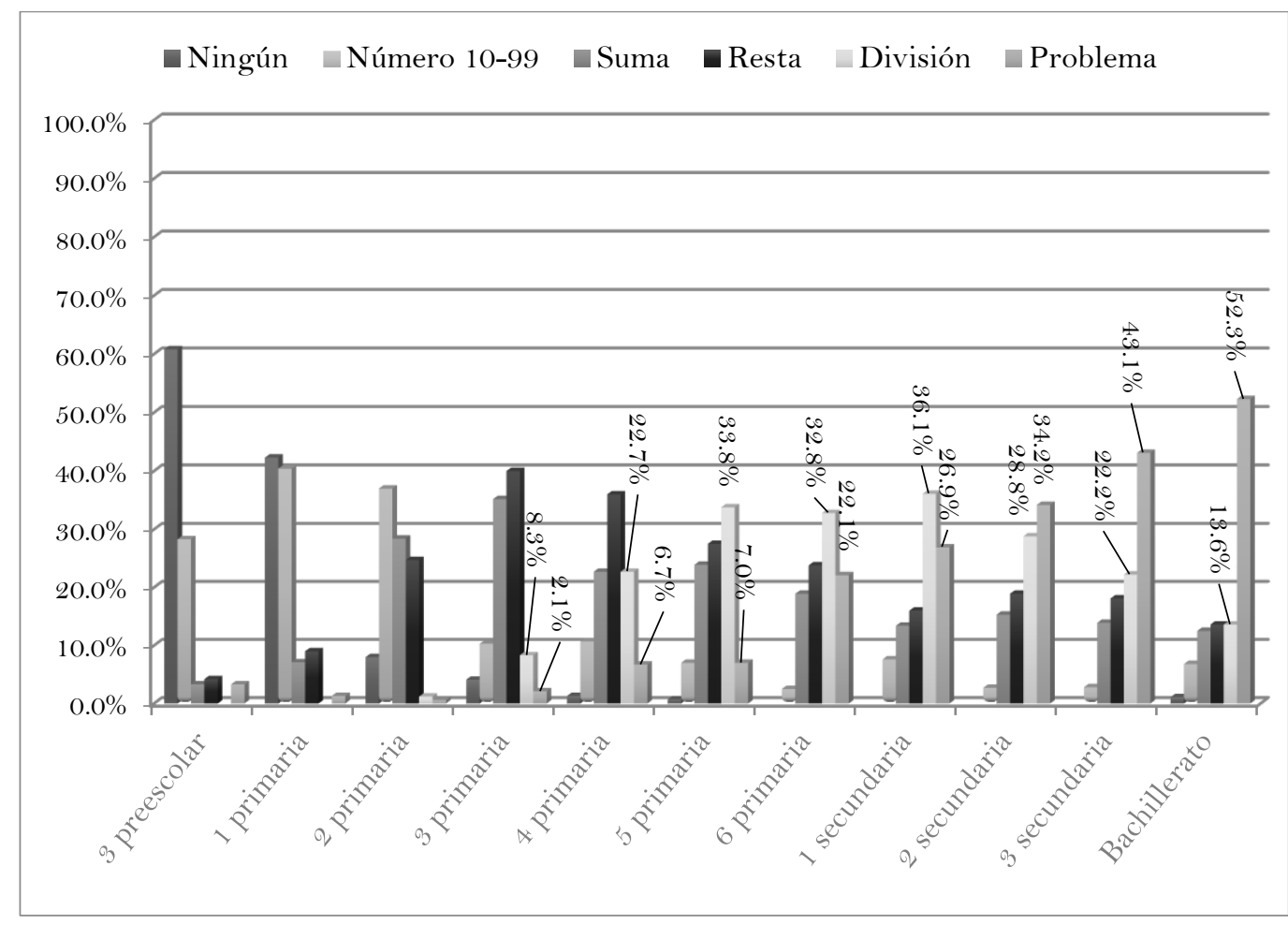

Figura 2. Porcentajes de aritmética por grado escolar, Yucatán Fuente: Elaboración propia. 
La tabla 8, con los valores acumulados, permite identificar además problemas para la resolución de restas y división. Así, solo el 50,4\% de tercero de primaria y el 68-3\% de sexto pudo resolver las restas, y solo el $54,9 \%$ de sexto de primaria y el $65,3 \%$ de tercero de secundaria pudo resolver divisiones correspondientes a cuarto grado.

Tabla 8. Porcentaje de sujetos que lograron hacer correctamente cada uno de los niveles de aritmética instrumento MIA. Yucatán

\begin{tabular}{|c|c|c|c|c|c|}
\hline & NÚMERO 10-99 & SUMA & RESTA & DIVISIÓN & PROBLEMA \\
\hline 3 preescolar & $39,1 \%$ & $10,8 \%$ & $7,5 \%$ & $3,3 \%$ & $3,3 \%$ \\
\hline 1 primaria & $57,8 \%$ & $17,4 \%$ & $10,3 \%$ & $1,3 \%$ & $1,3 \%$ \\
\hline 2 primaria & $91,9 \%$ & $54,9 \%$ & $26,5 \%$ & $1,8 \%$ & $0,6 \%$ \\
\hline 3 primaria & $95,9 \%$ & $85,6 \%$ & $50,4 \%$ & $10,4 \%$ & $2,1 \%$ \\
\hline 4 primaria & $98,8 \%$ & $88,1 \%$ & $65,4 \%$ & $29,4 \%$ & $6,7 \%$ \\
\hline 5 primaria & $99,2 \%$ & $92,2 \%$ & $68,3 \%$ & $40,8 \%$ & $7,0 \%$ \\
\hline 6 primaria & $100,1 \%$ & $97,6 \%$ & $78,7 \%$ & $54,9 \%$ & $22,1 \%$ \\
\hline 1 secundaria & $100,0 \%$ & $92,4 \%$ & $79,0 \%$ & $63,0 \%$ & $26,9 \%$ \\
\hline 2 secundaria & $99,9 \%$ & $97,2 \%$ & $81,9 \%$ & $63,0 \%$ & $34,2 \%$ \\
\hline 3 secundaria & $100,1 \%$ & $97,3 \%$ & $83,4 \%$ & $65,3 \%$ & $43,1 \%$ \\
\hline Bachillerato & $98,8 \%$ & $92,0 \%$ & $79,5 \%$ & $65,9 \%$ & $52,3 \%$ \\
\hline
\end{tabular}

Fuente: Elaboración propia sobre resultados de la investigación.

Estos datos permiten concluir que un porcentaje importante de niños y jóvenes en Yucatán presentan problemas en la adquisición de competencias básicas, específicamente, se identifican problemas de comprensión inferencial, resolución de problemas matemáticos básicos y uso de operaciones como la resta y la división.

\subsection{Factores asociados}

Respecto de los factores asociados a estos resultados, en esta sección identificamos tres tipos: individuales, familiares y sociales. Todos los análisis relacionados con lectura se realizaron con la muestra de los niños y niñas a partir de $3^{\circ}$ de primaria y de matemáticas a partir de $5^{\circ}$.

\subsubsection{Factores individuales}

Un primer conjunto de factores asociados a adquisición de competencias básicas tiene que ver con las características de los sujetos.

En esta investigación, contrario a lo que reporta la literatura (Carvallo Pontón, Caso y Contreras, 2007), utilizando la prueba t de Student y la U de Mann-Whitney, no se encontraron diferencias estadísticamente significativas en la parte de lectura de acuerdo al sexo: la media de las mujeres fue de 5,38 (DE=1,021) y la de los hombres de 5.39 $(\mathrm{DE}=1,042)$. En matemáticas, a pesar de observar diferencias aparentemente importantes en las medias, estas no resultaron significativas: la media de las mujeres fue de 4,65 (DE=1,227) y la de los hombres de 4,49 (DE=1,221).

Utilizando correlaciones de Spearman, la edad y el grado escolar se relacionan de manera positivo a moderada con lectura ( $\mathrm{rho}=, 700$ y ,714 sig al ,001) y matemáticas (rho=,675 y ,671 sig. al ,001). La correlación entre estas variables es un resultado esperado, dado que el proceso de maduración en el niño facilita el aprendizaje, y la asistencia a la escuela permite la adquisición de los aprendizajes básicos, sin embargo esta asociación no llega a ser alta. 
El segundo factor que mostró asociaciones significativas fue la motivación hacia la escuela. A los niños se les preguntó si creían que sirviera de algo asistir a la escuela. El $84,8 \%(\mathrm{~N}=1146)$ afirmó que "sirve mucho" ir a la escuela, en oposición al $0,8 \%(\mathrm{~N}=11)$ que respondió que "no sirve de nada". De igual forma, el 67,4\% afirmó que le "gusta mucho" asistir a la escuela, en oposición al 2,4\% que respondió "no me gusta". Las pruebas estadísticas mostraron correlaciones positivas, bajas pero significativas, entre la percepción que la escuela sirve y aprendizajes, tanto en lectura $(\mathrm{rho}=, 215$, sig. al ,001) como en aritmética (rho=,137 sig. al ,001). Este nivel de asociación, comparado con otras variables asociadas, es digno de resaltarse, primero por su significancia y fuerza estadística, pero sobretodo porque permite distinguir una dimensión vulnerable de modificación que podría estar precediendo al aprendizaje y que ha sido sub-analizada en las grandes evaluaciones estandarizadas.

Un tercer factor individual asociado al logro fue el apartado de discapacidad. Al respecto, llama la atención que el 25,3\% (N=354) de los padres refirieron que sus hijos o hijas presentaban al menos una condición de discapacidad (SEDESOL, 2014). La tabla 9 muestra valores agregados.

Tabla 9. Condiciones de discapacidad, valores agregados.

\begin{tabular}{lcc}
\hline & FRECUENCIA & PORCENTAJE \\
\hline $\begin{array}{l}\text { Dificultad para poner atención, aprender cosas sencillas o } \\
\quad \text { concentrarse }\end{array}$ & 217 & 46,3 \\
$\begin{array}{l}\text { Enfermedad crónica (corazón, cáncer, asma, artritis, otra) que le } \\
\quad \text { impida ir a la escuela }\end{array}$ & 87 & 18,6 \\
No puede ver bien & & 72 \\
No puede hablar o tiene dificultad para hacerlo & 45 & 15,4 \\
Tiene problemas para moverse & 19 & 9,6 \\
No puede oír & 15 & 4,1 \\
Tiene retraso o deficiencia mental & 14 & 3,2 \\
\hline Total & 469 & 100,0 \\
\hline Fur
\end{tabular}

Fuente: Elaboración propia con resultados de la investigación.

Casi la mitad de los padres refieren que sus hijos o hijas presentan dificultad para poner atención, aprender cosas sencillas o concentrarse. Este dato se complementa con el 12,6\% que, en el apartado siguiente de síntomas de salud mental (tabla 11), menciona que su hijo parece como retardado o lento para aprender. Este dato lleva a cuestionarse sobre varios aspectos: las causas por las que se reduce la atención, el manejo de las emociones, la incidencia de problemas de atención y aprendizaje, los métodos de enseñanza, etc. Y nuevamente pone sobre la mesa antecedentes del aprendizaje, modificables, que muestran relación negativa con los resultados de logro.

$\mathrm{Al}$ respecto, las condiciones de discapacidad tuvieron una correlación negativa con los resultados de logro identificados: en lectura rho $=-, 055$ al ,01 y en matemáticas rho $=-$ ,119 al,001.

Resultan significativas también las diferencias entre los que tienen al menos una de estas condiciones y los que no, en ambas partes del instrumento.

Muy relacionado con lo anterior, el cuarto factor individual fue la presencia de síntomas de problemas en la salud mental (Gómez Espinoza et al., 1993). En los datos recolectados, el 51,3\% afirmó que no tenía ninguna molestia, pero casi la mitad de los padres, refieren la presencia de al menos uno de los síntomas: el 23,3\% uno y el $25,4 \%$ 
más de uno. El síntoma que presentó más frecuencia fue "casi nunca juega con otros niños/as" con 21,3\% (tabla 11). Esto es que uno de cada cinco niños pudiera estar con problemas de sociabilización y esto a su vez estar afectando su desempeño escolar.

Tabla 10. Diferencias entre ausencia/presencia de condiciones de discapacidad

\begin{tabular}{lccccc}
\hline & CONDICIONES DE DISCAPACIDAD & N & MEDIA & DE & T \\
\hline \multirow{2}{*}{ Lectura } & Ausencia & 1035 & 4,62 & 1,698 & \multirow{2}{*}{$2,353^{*}$} \\
\hline \multirow{2}{*}{ Aritmética } & Presencia & 350 & 4,35 & 1,888 & \\
& Ausencia & 1043 & 3,72 & 1,586 & \multirow{2}{*}{$4,309^{* * *}$} \\
\hline
\end{tabular}

Fuente: Elaboración propia con resultados de la investigación (*sig. al ,05; ***sig. al ,001).

Tabla 11. Síntomas de problemas de salud mental. Valores agregados. Yucatán

\begin{tabular}{|c|c|c|}
\hline MOLESTIAS QUE SU HIJO/A PUDO HABER PRESENTADO & FRECUENCIA & PoRCEnTAJE \\
\hline ¿Casi nunca juega con otros niños/as? & 271 & 21,3 \\
\hline ¿El lenguaje del niño/a es anormal en alguna forma? & 217 & 17,0 \\
\hline ¿Se asusta o se pone nervioso sin razón? & 189 & 14,8 \\
\hline ¿Sufre el niño/a de dolores de cabeza? & 177 & 13,9 \\
\hline ¿Parece como retardado o lento para aprender? & 160 & 12,6 \\
\hline ¿El niño/a duerme mal? & 83 & 6,5 \\
\hline ¿El niño/a se orina o defeca en la ropa? & 71 & 5,6 \\
\hline $\begin{array}{l}\text { ¿Ha tenido el niño/a en algunas ocasiones convulsiones o caídas al } \\
\text { suelo sin razón? }\end{array}$ & 40 & 3,1 \\
\hline ¿Ha robado cosas de la casa? & 35 & 2,7 \\
\hline ¿El niño/a ha huido de la casa frecuentemente? & 31 & 2,4 \\
\hline Total & 1274 & 100,0 \\
\hline
\end{tabular}

Fuente: Elaboración propia con resultados de la investigación.

Las correlaciones entre los aprendizajes básicos y los síntomas de problemas de salud mental y de conducta resultan negativas, muy bajas pero significativas para ambas pruebas: en lectura rho $=-, 065$, sig. al ,01 y en aritmética: rho $=-, 112$, sig. al ,001. Y tener al menos un síntoma afecta significativamente los resultados en lectura y matemáticas (tabla 12).

Tabla 12. Comparación entre grupos con ausencia y presencia de síntomas de problemas de salud mental

\begin{tabular}{|c|c|c|c|c|c|}
\hline & $\begin{array}{c}\text { SÍNTOMAS DE PROBLEMAS DE } \\
\text { SALUD MENTAL }\end{array}$ & $\mathbf{N}$ & MEDIA & $\overline{D E}$ & $\bar{T}$ \\
\hline \multirow{2}{*}{ Lectura } & Ausencia & 710 & 4,69 & 1,641 & \multirow{2}{*}{$2.996^{*}$} \\
\hline & Presencia al menos de una & 674 & 4,41 & 1,847 & \\
\hline \multirow{2}{*}{ Aritmética } & Ausencia & 716 & 3,79 & 1,538 & \multirow{2}{*}{$4.338^{* * * *}$} \\
\hline & Presencia al menos de una & 680 & 3,43 & 1,598 & \\
\hline
\end{tabular}

Fuente: Elaboración propia con resultados de la investigación (*sig. al ,05; *** sig. al 0,001).

La diferencia en la sumatoria de síntomas de problemas de salud mental entre los diferentes niveles MIA lectura resulta significativa $(\mathrm{F}=5,025 \mathrm{y}$ sig. al ,001). En los niveles más bajos de lectura, se observan medias más elevadas de síntomas psiquiátricos (figura 3). 
En matemáticas a pesar de que las diferencias no son significativas, los participantes que llegan al nivel problema presentan una media menor que los que se quedan en número (figura 4).

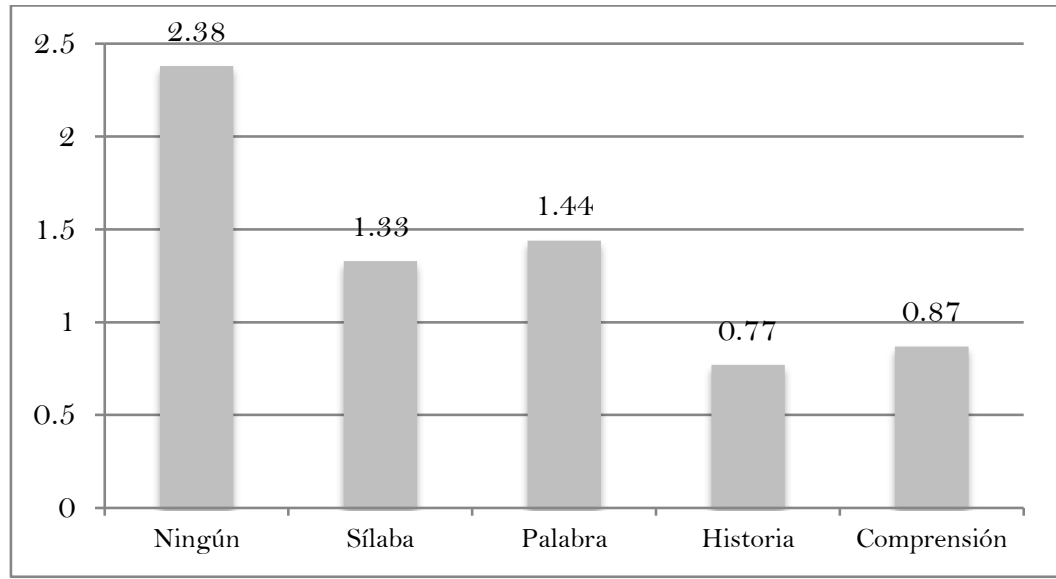

Figura 3. Medias de síntomas de problemas de salud mental por nivel alcanzado MIA lectura.

Fuente: Elaboración propia.

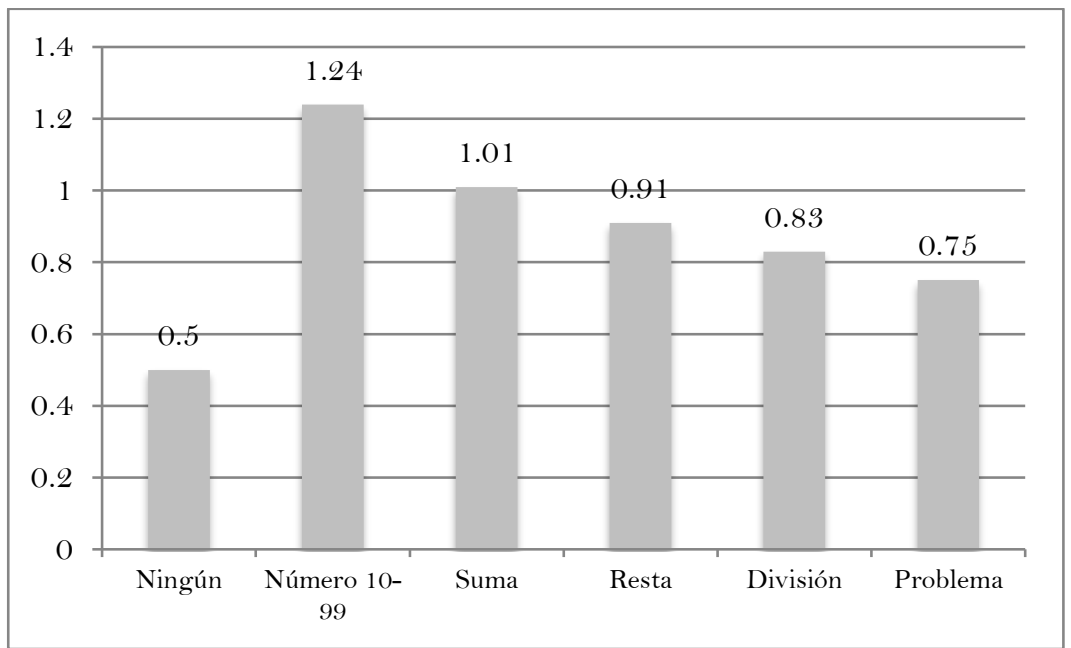

Figura 4. Medias de síntomas de problemas de salud mental por nivel en MIA matemáticas.

Fuente: Elaboración propia.

Estos datos al igual que los relacionados al apartado anterior de condiciones de discapacidad, remiten a cuestiones psicológicas, sociales y familiares que podrían ser abordadas.

\subsubsection{Factores familiares: capital cultural escolar y condiciones económicas del hogar}

Un segundo tipo de factores asociados al logro tienen que ver con ciertas características de las familias y los hogares, en particular en relación con los factores ampliamente 
estudiados de capital cultural escolar y condiciones económicas, como el acceso a servicios o número de cuartos.

Respecto del capital cultural escolar, este incorpora la escolaridad y ocupación de los padres, las expectativas de escolaridad de sus hijos, y el acceso a ciertos bienes y servicios como el número de libros en el hogar, acceso a internet o posibilidades de asistir al cine.

En esta investigación, la edad promedio de la madre fue de 37,8 años (DE: 13,801) y del padre 44,3 años $(\mathrm{DE}=19,984)$. En estos casos, la edad de la madre y del padre se relaciona de forma positiva, baja, pero significativa con los resultados en los aprendizajes básicos de ambas pruebas.

Tabla 13. Relaciones entre edad padres y resultados logro alcanzado

\begin{tabular}{lcc}
\hline & EDAD MADRE & EDAD PADRE \\
\hline Lectura &, $239^{* * *}$ &, $181^{* * * *}$ \\
Aritmética &, $213^{* * *}$ &, $165^{* * * *}$ \\
\hline
\end{tabular}

Fuente: Elaboración propia con resultados de la investigación (***sig. al 0,001).

En términos de ocupación principal, el 94,9\% de las madres se ocupaban de labores del hogar, seguido de trabajadoras en servicios y vendedores en comercio, con $8,4 \%$. En el caso de los padres, el $23,7 \%$ trabajaba en ocupaciones elementales como peones, jornaleros, ambulantes o albañiles, seguido de trabajadores de servicios con un $17,4 \%$ y de obreros y operarios, con un 10,7\%.

En términos de escolaridad, la moda y mediana tanto del padre como de la madre es 3ro de secundaria (28,2\% para el caso de las madres y $22,8 \%$ para el caso de los padres), donde un 4,3\% en promedio nunca asistió a la escuela. Aquí se encuentran correlaciones bajas pero significativas solo entre los resultados de aritmética y la escolaridad de la madre (rho=,094 sig. al ,001) y del padre (rho=,115, sig. al ,001).

En relación con las expectativas de escolaridad, el 54,8\% espera que su hijo/a llegue a posgrado y el $18,8 \%$ a carrera técnica. Por último, solo el 30,6\% de los hogares afirmó tener internet, el 44,7\% no asiste nunca al cine y el $18,5 \%$ solo una vez al año, en oposición al 6,1\% que afirmó asistir varias veces al mes, y en el 36,5\% de los hogares cuentan entre 11 y 50 libros. Todas las variables se relacionan de forma positiva, baja pero significativa con aritmética, y los libros en el hogar y las expectativas de estudio además con lectura (tabla 14).

Tabla 14. Correlaciones entre acceso a bienes culturales y logro alcanzado

\begin{tabular}{lcc}
\hline & LECTURA & ARITMÉTICA \\
\hline Internet & $\mathrm{NS}$ &, $116^{* * * *}$ \\
Libros en el hogar &, $134^{* * *}$ &, $105^{* * *}$ \\
Asistencia al cine & $\mathrm{NS}$ &, $127^{* * *}$ \\
Expectativas de estudio &, $126^{* * *}$ &, $141^{* * *}$
\end{tabular}

Fuente: Elaboración propia con resultados de la investigación (***sig. al 0,001)

Respecto de las condiciones económicas del hogar, el 98,9\% tenían acceso a agua y el $79,8 \%$ a drenaje. La media de cuartos en el hogar fue de $2,30(\mathrm{DE}=1,101)$ y en promedio vivían 3,69 (DE=10,651) niños por hogar. En este caso, las diferencias entre los que 
tienen drenaje y no, resultaron significativas para ambos apartados. Se muestran las medias que son más elevadas en el grupo que sí cuenta con el servicio.

Tabla 15. Relación entre drenaje y logro alcanzado

\begin{tabular}{lccccc}
\hline & & N & MEDIA & DE & T \\
\hline \multirow{2}{*}{ Lectura } & si & 1083 & 4,63 & 1,720 & \multirow{2}{*}{$2,912^{* *}$} \\
Aritmética & no & 273 & 4,26 & 1,860 & \\
& si & 1092 & 3,65 & 1,565 & \multirow{2}{*}{$2,490^{*}$} \\
\hline
\end{tabular}

Fuente: Elaboración propia con resultados de la investigación (*sig. al ,05; **sig. al 0,01).

De igual manera, las correlaciones entre el número de cuartos y ambas partes del instrumento resultan muy bajas, pero significativas: $r h o=0,84$ sig. al 0,01 para lectura y rho $=0,160$ sig. al 0,01 para aritmética.

\subsubsection{Factores sociales y culturales: lengua materna y acceso a bibliotecas}

Por último, y en relación cercana con el nivel socioeconómico, se encontraron algunas asociaciones con factores sociales y culturales, en particular con la lengua materna que se habla en el hogar. Así, el 39,1\% de los hogares afirmaron hablar una lengua originaria, mayoritariamente maya. Como se muestra en la siguiente tabla, usando una prueba $\mathrm{T}$ se encontraron diferencias significativas en aritmética, pero no en lectura.

Tabla 16. Relación entre lengua originaria y logro alcanzado

\begin{tabular}{lccccc}
\hline & HABLAN LENGUA INDÍGENA & N & MEDIA & DE & T \\
\hline \multirow{2}{*}{ Lectura } & Sí & 584 & 4,51 & 1,784 & \multirow{2}{*}{ NS } \\
\multirow{2}{*}{ Aritmética } & No & 764 & 4,60 & 1,732 & \\
& Sí & 587 & 3,42 & 1,528 & \multirow{2}{*}{$3,846^{* * *}$} \\
\hline
\end{tabular}

Fuente: Elaboración propia con resultados de la investigación (***sig. al 0,001).

Tabla 17. Relación entre existencia de biblioteca y logro alcanzado

\begin{tabular}{lccccc}
\hline & BIBLIOTECA EN COLONIA O LOCALIDAD & N & MEDIA & DE & F \\
\hline \multirow{3}{*}{ Lectura } & Sí & 430 & 4,72 & 1,648 & \\
& No & 813 & 4,55 & 1,772 & $4,212^{* *}$ \\
\multirow{4}{*}{ Matemáticá } & No sé & 123 & 4,01 & 1,835 & \\
& Sí & 433 & 3,79 & 1,543 & \\
& No & 822 & 3,55 & 1,580 & $2,922^{*}$ \\
\hline \multirow{2}{*}{ Lectura } & No sé & 123 & 3,37 & 1,611 & \\
& BIBLIOTECA EN ESCUELA & $\mathbf{N}$ & MEDIA & & F \\
& Sí & 1153 & 4,67 & 1,668 & \\
Matemáticas & No & 166 & 3,91 & 2,074 & $14,743^{* * *}$ \\
& No sé & 39 & 3,51 & 1,890 & \\
& Sí & 1162 & 3,70 & 1,532 & \\
& No & 169 & 3,14 & 1,754 & $10,020^{* * * *}$ \\
\hline
\end{tabular}

Fuente: Elaboración propia con resultados de la investigación (*sig. al ,05; **sig. al 0,01; ***sig. al 0,001$)$.

Otro factor social analizado fue el acceso a bibliotecas públicas y escolares. Respecto a la biblioteca pública, solo el 31,4\% ( $\mathrm{N}=433)$ participantes afirmó que sí existía biblioteca en su localidad o colonia, y solo el 4,1\% (N=54) afirmó que asistía "frecuentemente" o "muy frecuentemente a la misma”. En cambio, el 84,7\% afirmó que su escuela tenía biblioteca y 
el 47\% afirmó que asistía "de vez en cuando", el 15\% "frecuentemente" y el 9,5\% "muy frecuentemente". En la siguiente tabla se encuentran diferencias significativas en las capacidades básicas de acuerdo a la presencia de biblioteca pública en el lugar en el que viven, y la biblioteca escolar, en ambas pruebas.

Y como se puede inferir, mientras más utilicen ambas bibliotecas tienen mejores niveles de logro alcanzado.

Tabla 18. Relación entre asistencia y uso biblioteca y logro alcanzado

\begin{tabular}{lcc}
\hline & LECTURA & MATEMÁTICAS \\
\hline Asistes a la biblioteca pública &, $104^{* * * *}$ &, $114^{* * *}$ \\
Utilizas libros de la biblioteca de tu escuela &, $109^{* * * *}$ &, $092^{* * *}$ \\
\hline
\end{tabular}

Fuente: elaboración propia con resultados de la investigación (****sig. al 0.001).

El uso que se da de las bibliotecas y su relación con el logro educativo merece ser abordado con mayor detenimiento, tanto por su poder de propagación, como por su potencialidades.

\section{Discusión y conclusiones}

Los resultados presentados muestran una serie de déficit en la adquisición de capacidades básicas a lo largo de la trayectoria escolar de niñas/os y adolescentes en Yucatán. Si bien la asistencia a la escuela es fundamental para aprender a leer y resolver operaciones matemáticas básicas, los resultados sugieren que los estudiantes no están adquiriendo estas competencias básicas en los primeros años escolares, y se infiere que pueden ir arrastrando a lo largo de su trayectoria educativa estos problemas, afectando su desempeño en la escuela.

Llama la atención los altos porcentajes de algunas condiciones de discapacidad y síntomas de salud mental y su relación negativa con los aprendizajes; la asociación entre la motivación por la escuela y los resultados en MIA y; la importancia de la existencia y uso de la biblioteca escolar y municipal.

Desde la perspectiva constructivista el aprendizaje es un asunto que implica procesos mentales y funciones cognitivas, evaluación cognitiva basada en compromisos y creencias, pero también aspectos sociales y mediación cultural (Ausubel, 2002; Coll, Palacios y Marchesi, 2010; Hernández, 2012). Así, las explicaciones que dan cuenta de las diferencias individuales en los resultados del logro académico en diferentes niveles van más allá de las habilidades cognitivas, y se han dirigido hacia variables relacionadas al contexto social y escolar. Se considera que es necesario poner énfasis en los factores individuales que anteceden al aprendizaje y funcionan como precursores para que se den los procesos de asimilación.

Las condiciones económicas mantienen la supremacía de los factores asociados ya que se relacionan de manera importante con los resultados de logro educativo, pero además se encuentran implicadas en la mayoría de las variables asociadas. Dentro de esta investigación, el capital cultural (escolaridad de los padres, acceso a internet, asistencia a eventos culturales, expectativas de estudio), el hablar una lengua indígena, la edad de los padres y la presencia de biblioteca en su escuela o en su comunidad son variables que implican y se ven modificadas por las variables económicas. Sin embargo, aspectos como 
el uso que se da de las bibliotecas, la motivación hacia la escuela, síntomas de salud mental y las condiciones de discapacidad son variables que al mostrar relación con los resultados en los aprendizajes básicos se vislumbran como caminos aún no del todo explorados que pueden dar luz a la comprensión del tema.

Los resultados que se presentan en relación a las deficiencias en los aprendizajes básicos de los niños y niñas dan pautas específicas que guían la acción, ya que permiten focalizar y dirigir esfuerzos a tareas y contenidos específicos, además de que se reportan de manera sencilla. Por su parte, los resultados referentes a los factores asociados aportan a la comprensión de las variaciones individuales de estos resultados de aprendizajes básicos, pero sobretodo permiten resaltar aspectos susceptibles de modificación e intervención.

De esto se pueden inferir dos grandes tipos de conclusión, la primera relacionada con orientaciones de política educativa respecto de los resultados encontrados y la segunda más orientada a los procesos mismos de evaluación educativa.

Respecto del primer punto, los resultados sugieren que es necesario implementar políticas y programas educativos específicos que fortalezcan estas competencias en los primeros ciclos de primaria. Los resultados respecto a lectura muestran con mayor claridad que es en $1^{\circ}$ y $2^{\circ}$ de primaria donde la mayoría de los niños yucatecos incrementan sus capacidades lectoras. Este salto no se aprecia con la misma claridad en las operaciones aritméticas: la identificación de números y la resolución de sumas y restas no se adquieren con la misma velocidad, ni en la misma proporción en estos años. De igual forma, se necesitan intervenciones específicas en los últimos años de primaria y en secundaria que trabajen con aquellos niños que no logran adquirir estas competencias a lo largo de su trayectoria escolar. Los resultados mostraron que un porcentaje importante de sujetos que cursan secundaria tienen problemas de comprensión y de cálculo básico que además dificulta que sigan adquiriendo los aprendizajes esperados para el año que están cursando, por lo que es necesario generar acciones remediales o complementarias en estos grupos de edades para fortalecer sus capacidades básicas.

Como los resultados sugieren, para que estas políticas sean efectivas, requieren integrar en sus acciones elementos que disminuyan el efecto de las desigualdades individuales, familiares y sociales aquí identificadas. La antropología social en México ha estudiado los procesos de "acumulación de desventajas", que remiten al efecto negativo y acumulativo de condiciones en las familias que limitan su desarrollo (González de la Rocha, 2006). Por ello, es necesario incluir acciones que atiendan la prevalencia de molestias psiquiátricas y condiciones de discapacidad, que mejoren la sociabilidad de los niños y niñas, y que utilicen la infraestructura educativa, en particular las bibliotecas públicas y escolares para incrementar la motivación y autoeficacia.

De igual manera, los resultados sugieren la necesidad de implementar acciones específicas relacionadas con aritmética. Aprovechando el contexto de discusión sobre el nuevo modelo educativo, es necesario asegurar que la enseñanza de las matemáticas, y de la aritmética en particular, resulte pertinente y relevante para los estudiantes, y que se generen aprendizajes significativos que permitan subsanar los déficits identificados en esta investigación y mejorar los resultados de logro educativo en esta área del conocimiento. 
Respecto del segundo punto, se puede concluir que la implementación de estas evaluaciones ciudadanas -realizada en hogares con instrumentos simples que aplican voluntarios a todos los niños/as- abren una agenda de investigación que permitan pensar su relevancia y pertinencia respecto de diversas evaluaciones alternativas que están demostrando un potencial enorme. Como otros autores han discutido (Aboites, 2012; Monereo, 2009; Perrenoud, 2008) existen importantes efectos de la evaluación en la escuela y en el proceso de enseñanza-aprendizaje y es muy difícil pensar que un solo instrumento o procedimiento para medir competencias es suficiente. La evaluación auténtica (Monereo, 2009), la evaluación dinámica (Sternberg y Grigorenko, 2003), y la utilización de las tareas escolares (Monereo, 2003) son algunos ejemplos de cómo se pueden pensar evaluaciones alternativas. En este sentido, la agenda de investigación requiere ponderar cómo se pueden articular procesos de evaluación dinámica con estas evaluaciones ciudadanas en diversas partes del mundo. La medición y evaluación de competencias básicas y factores asociados, usando evaluaciones ciudadanas como las que generaron la información aquí analizada han resultado ser una innovación educativa costo-efectiva que, a la par que complementa las evaluaciones gubernamentales, permite involucrar a más población en la educación y generar conocimiento que permita transformar nuestra realidad (Levine, 2015), pero que requieren comprender más cómo la suma de diversas aproximaciones permitan entender mejor cómo las competencias básicas aquí analizadas se desarrollan dentro y fuera del sistema escolar.

\section{Referencias}

Aboites, H. (2012). La medida de una nación: Los primeros años de la evaluación en México: Historia de poder, resistencia y alternativa (1982-2012). Ciudad de México: CLACSO.

Ausubel, D. (2002). Adquisición y retención del conocimiento. Una perspectiva cognitiva. Barcelona: Paidós.

Ames, C. (1992). Classroom: Goals, structures and student motivation. Journal of Educational Psychology, 84(3), 261-271.

Azuara, P. y Reyes, I. (2011). Negotiating worlds: A young Mayan child developing literacy at home and at school in Mexico. Compare: A Journal of Comparative and International Education, 41(2), 181-194.

Backhoff, E. (2011). La inequidad educativa en México: Diferencias en el aprendizaje de la comprensión lectora en educación básica. Profesorado. Revista de Currículum y Formación de Profesorado, 15(3), 87-102.

Backhoff, E., Bouzas, A., Contreras, C., Hernández, E. y García, M. (2007). Factores escolares y aprendizaje en México. El caso de la educación básica. Ciudad de México: INEE.

Backhoff, E. y Contreras Roldán, S. (2014). “Corrupción de la medida” e inflación de los resultados de ENLACE. Revista Mexicana de Investigación Educativa, 19(63), 1267-1283.

Banerji, R., Bhattacharjea, S. y Wadhwa, W. (2013). The Annual Status of Education Report (ASER). Research in Comparative and International Education, 8(3), 387. https://doi.org/10.2304/rcie.2013.8.3.387

Barroso-Tanoira, F. G. (2014). Motivos para la baja voluntaria definitiva de alumnos de licenciatura en instituciones de educación superior privadas: Un estudio en el sureste de México. Revista Iberoamericana de Educación Superior, 5(14), 19-40. 
Blanco, E. (2013). Los límites de la escuela. Educación, desigualdad y aprendizajes en México. Ciudad de México: El Colegio de México.

Bravo-Sanzana, M. y Salvo, S. (2015). La importancia de la salud emocional en la escuela, un factor a considerar para la mejora de la salud mental y los logros en el aprendizaje. Salud Pública de México, 57(2), 111-112.

Carvallo Pontón, M., Caso, J. y Contreras, L. Á. (2007). Estimación del efecto de variables contextuales en el logro académico de estudiantes de Baja California. REDIE. Revista Electrónica de Investigación Educativa, 9(2).

Cervini, R. y Dari, N. (2009). Género, escuela y logro escolar en matemática y lengua de la educación media: Eestudio exploratorio basado en un modelo multinivel bivariado. Revista Mexicana de Investigación Educativa, 14(43), 1051-1078.

Coleman, J. S., Campell, E. Q., Hobson, C. J., Mood, A. M., Weinfeld, F. y York, R. (1966). Equality of educational opportunity. Washington D. C.: US Deparment of Health, Education \& Welfare. Office of Education.

Coll, C., Palacios, J. y Marchesi, A. (2010). Desarrollo psicológico y educación. 2. Psicología de la educación escolar. Madrid: Alianza.

CONEVAL. (2015). Medición de la pobreza en México y en las Entidades Federativas. 2014. México: CONEVAL. Recuperado de

http://web.coneval.gob.mx/coordinacion/entidades/Documents/Veracruz/principal/30i nforme2012.pdf

Couoh Lope, C. L., Góngora, A., García Rivero, A., Macías Aguilar, I. R. y Olmos Barragán, N. A. (2015). Ansiedad y autoestima en escolares de educación primaria de Mérida, Yucatán. Enseñanza e Investigación en Psicología, 20(3), 302-308.

Duru-Bellat, M. (2004). Social inequality at school and educational policies. Paris: IIEP; UNESCO. Recuperado de http://publications.iiep.unesco.org/Social-inequality-school-educationalpolicies

Dutt, S. C., Kwauk, C. y Robinson, J. P. (2016). Pratham's read India program. Taking small steps toward learning at scale. Washington D. C.: Center for Universal Education. Brookings Institution.

Gómez Espinoza, M., Rico Diaz, H., Caraveo Anduaga, J. y Guerrero Cansino, G. (1993). Validez de un instrumento de tamizaje (rqc). Anales-Instituto Mexicano de Psiquiatría, 4, 204-208.

González de la Rocha, M. (2006). Espirales de desventajas: Pobreza, ciclo vital y aislamiento social. En G. Saraví (Ed.), De la pobreza a la exclusión: Continuidades y rupturas de la cuestión social en América Latina (pp. 137-166). Ciudad de México: Prometeo Libros.

Hanushek, E. A. y Luque, J. A. (2002). Efficiency and equity in schools around the world (working paper No. 8949). Cambridge, MA: National Bureau of Economic Research.

Hernández, G. (2012). Miradas constructivistas en psicología de la educación. Ciudad de México: Paidós.

Hernández Padilla, E. y González, M. J. (2011). Modelo de ecuación estructural que evalúa las relaciones entre el estatus cultural y económico del estudiante y el logro educativo. Revista Electrónica de Investigación Educativa, 13(2), 188-203.

Hernández, R., Fernández, C. y Baptista, P. (2001). Metodología de la investigación. Ciudad de México: McGraw-Hill. 
Hevia, F. J. y Vergara-Lope, S. (2016). Evaluaciones educativas realizadas por ciudadanos en México: Validación de la Medición Independiente de Aprendizajes. Innovación Educativa, 16(70), 85-110.

INEE. (2013). El aprendizaje en sexto de primaria en México. Informe sobre los resultados del Excale 06, aplicación 2009. Español, matemáticas, ciencias naturales y educación cívica. Ciudad de México: Autor.

INEE. (2015). Plan Nacional para la Evaluación de los Aprendizajes (PLANEA). Resultados nacionales 2015. $6^{\circ}$ de primaria y $3^{\circ}$ de secundaria. Lenguaje y comunicación. Matemáticas. Ciudad de México: Autor.

INEE. (2016a). El aprendizaje en tercero de secundaria en México. Informe de resultados. Excale o9 aplicación 2012. Español, matemáticas, ciencias y formación cívica y ética. Ciudad de México: Autor.

INEE. (2016b). Panorama educativo en México. Indicadores del Sistema Educativo Nacional 2015. Educación básica y media superior. Ciudad de México: Autor.

INEGI. (2016). Yucatán. Recuperado de http://cuentame.inegi.org.mx/monografias/informacion/yuc/default.aspx?tema=me\&e= 31

Instituto de Estadística de la UNESCO. (2016). Evaluación Oral de la Lectura: Recomendaciones de donantes, implementadores y profesionales. Montreal: Autor.

Jiménez, V. y Valle, R. (julio, 2013). Factores de salud asociados al desempeño escolar: seguimiento de una generación del bachillerato en la UNAM. Comunicación presentada en la Tercera Conferencia Latinoamericana sobre el abandono en la Educación Superior, Ciudad de México.

Kerlinger, F. N. y Lee, H. B. (2002). Investigación del comportamiento: Método de investigación en ciencias sociales. Ciudad de México: McGraw-Hill.

Levine, R. (2015). Friday note: Making the movement for accountability and learning. Recuperado de http://www.hewlett.org/blog/posts/friday-note-making-movement-accountability-andlearning

Martínez Rizo, F. (2009). Evaluación formativa en aula y evaluación a gran escala: Hacia un sistema más equilibrado. Revista Electrónica de Investigación Educativa, 11(22). Recuperado de http://redie.uabc.mx/vol11 no2/contenido-mtzrizo2.html

McLauchlan, P. (2009). Difusión y uso de resultados de evaluaciones educativas a gran escala en América Latina. En E. Martín y F. Martínez Rizo (Eds.), Avances y desafíos en la evaluación educativa (pp. 147-160). Madrid: Organización de Estados Iberoamericanos para la Educación, la Ciencia y la Cultura.

Méndez-González, R. M. (2010). Condiciones de vida y salud en zonas indígenas de Yucatán, México: 1990 y 2005. Población y Salud en Mesoamérica, 8(1), 1-18.

Mier y Terán, M. y Rabell, C. (2013). Escolaridad y lengua hablada en comunidades rurales de la península yucateca. Revista Mexicana de Sociología, 75(3), 371-406.

Mijangos, J. C., Soberanis, C. y Negrón, J. A. (2011). Idioma maya y currículo intercultural para estudiantes de primaria en Yucatán-México. Cuadernos Interculturales, 9(17), 199-213.

Mijangos-Noh, J. C. y Cardos-Dzul, M. P. (2011). The Mayas of Yucatan, Mexico: Their fight against school dropout. Journal of American Indian Education, 50(3), 61-79. 
Monereo, C. (2003). La evaluación del conocimiento estratégico a través de tareas auténticas. Pensamiento educativo, 32, 71-89.

Monereo, C. (2009). La autenticidad en la evaluación. En M. Castelló (Ed.), La evaluación auténtica en enseñanza secundaria y universitaria (pp. 9-24). Barcelona: Ebedé.

Montero, I. y León, O. (2007). A guide for naming research studies in Psychology. International Journal of Clinical and Health Psychology, 7(3), 847-862.

Perrenoud, P. (2008). La evaluación de los alumnos: De la producción de la excelencia a la regulación de los aprendizajes. Entre dos lógicas. Buenos Aires: Ediciones Colihue.

PAL Network. (2015). People's Action for Learning Network. Recuperado de http://palnetwork.org/?lang=es

PNUD. (2015). Objetivos de Desarrollo del Milenio-UNDP. Recuperado de http://www.undp.org/content/undp/es/home/mdgoverview/

Report, G. E. M. (2016). The PAL Network learning journey: Beyond assessment to action! Recuperado de https://efareport.wordpress.com/2016/02/18/the-pal-network-learning-journeybeyond-assessment-to-action/

Rocha, C. C. y Espejel, M. M. P. (2008). Perfil del maltrato (bullying) entre estudiantes de secundaria en la ciudad de Mérida, Yucatán. Revista Mexicana de Investigación Educativa, $13(38), 825-842$.

Save the Children. (2013). The right to learn. Community participation in improving learning. Westport: Autor.

SEDESOL. (2014). Encuesta de características socioeconómicas de los hogares, 2014 (ENCASEH). Ciudad de México: Autor.

SEGEY. (2016). SEGEY-Estadísticas. Recuperado de http://www.educacion.yucatan.gob.mx/estadistica/

SEP. (2011). Evaluación Nacional del Logro Académico en Centros Escolares, ENLACE. Recuperado de http://www.enlace.sep.gob.mx/

SEP. (2016a). El modelo educativo 2016. El planteamiento pedagógico de la Reforma Educativa. Ciudad de México: Secretaría de Educación Pública.

SEP. (2016b). ENLACE 2013. Recuperado de http://www.enlace.sep.gob.mx/content/gr/docs/2013/historico/04_EB_2013.pdf

Sima, E. G., Perales, M. D. y Be Ramírez, P. A. (2014). Actitudes de yucatecos bilingües de maya y español hacia la lengua maya y sus hablantes en Mérida, Yucatán. Estudios de Cultura Maya, XLIII, 157-179.

Stearns, R. D. (1986). Using ethnography to link school and community in rural Yucatan. $\begin{array}{lllll}\text { Anthropology } \quad \text { E } & \text { Education } & \text { Quarterly, } & \text { 17(1), }\end{array}$ https://doi.org/10.1525/aeq.1986.17.1.05x0976n

Sternberg, R. J. y Grigorenko, E. L. (2003). Evaluación dinámica: Naturaleza y evaluación del potencial de aprendizaje. Madrid: Grupo Planeta.

UNESCO. (1990). Declaración Mundial sobre Educación para Todos y Marco de Acción para Satisfacer las Necesidades Básicas de Aprendizaje. Jomtien: Autor.

UNESCO. (2015). Informe de resultados. Tercer estudio regional comparativo y explicativo. Factores asociados. Santiago de Chile: Autor. 
Zenorini, R. da P. C., Santos, A. A. A. y Monteiro, R. de M. (2011). Motivação para aprender: Relação com o desempenho de estudantes. Paidéia (Ribeirão Preto), 21(49), 157-164. https://doi.org/10.1590/S0103-863X2011000200003

\section{Breve CV de los autores}

\section{Samana Vergara-Lope Tristán}

Licenciada, Maestra y Doctora en Psicología por la Facultad de Psicología de la Universidad Nacional Autónoma de México (UNAM). Ha impartido cátedra, participado en comités de evaluación y dirigido múltiples tesis a nivel licenciatura y posgrado en diferentes instituciones e instancias de educación superior. Ha participado en la creación y validación de alrededor de 20 instrumentos de medición. Entre sus publicaciones se encuentran dos libros y varios capítulos de libros y artículos en revistas científicas arbitradas. Actualmente es Profesora de Tiempo Completo de la Universidad Veracruzana, coordinadora del proyecto Medición Independiente de Aprendizajes (MIA) y miembro del Sistema Nacional de Investigadores. ORCID ID: O000-0001-8029-3533. Email: samanavergaralope@hotmail.com

\section{Felipe J. Hevia}

Doctor en antropología por CIESAS-Centro de Investigaciones y Estudios Superiores en Antropología Social, donde es profesor investigador. Sus líneas de investigación son participación ciudadana, educación, contraloría social y políticas de combate a la pobreza. En 2015 recibió el Premio de Investigación en Ciencias Sociales por parte de la Academia Mexicana de Ciencias. Sus investigaciones han sido premiadas por la Universidad de Luxemburgo, el consorcio RISC, el PNUD, el BID, el gobierno de Brasil y el Consejo Latinoamericano de Administración-CLAD. Es coordinador del proyecto Medición Independiente de Aprendizajes, primera experiencia de evaluaciones ciudadanas de aprendizajes en México y América Latina. Es miembro del Sistema Nacional de Investigadores, nivel II. ORCID ID: O000-0002-4424-5320. Email: fhevia@ciesas.edu.mx

\section{Víctor Rabay Mora}

Estudiante de la carrera de psicología en la Facultad de Psicología de la Universidad Veracruzana, también estudiante de pedagogía en el sistema de enseñanza abierto de la Facultad de Pedagogía por la misma Universidad. Participante en el plan de desarrollo académico de la Facultad de psicología como representante de los alumnos. Actualmente, consejero suplente de la Facultad de psicología. Colaborador del proyecto Medición Independiente de Aprendizajes del Centro de Investigaciones y Estudios Superiores en Antropología Social y la Universidad Veracruzana. ORCID ID: 0000-0002-4227-9068 Email: victorrabay19@hotmail.com 